\title{
Perovskite light-emitting diodes based on solution-processed self-organized multiple quantum wells
}

Nana Wang, Lu Cheng, Rui Ge, Shuting Zhang, Yanfeng Miao, Wei Zou, Chang Yi, Yan Sun, Yu Cao, Rong Yang, Yingqiang Wei, Qiang Guo, You Ke, Maotao Yu, Yizheng Jin, Yang Liu, Qingqing Ding, Dawei Di, Le Yang, Guichuan Xing, He Tian, Chuanhong Jin, Feng Gao, Richard H. Friend, Jianpu Wang and Wei Huang

\section{Journal Article}

\section{Tweet}

N.B.: When citing this work, cite the original article.

Original Publication:

Nana Wang, Lu Cheng, Rui Ge, Shuting Zhang, Yanfeng Miao, Wei Zou, Chang Yi, Yan Sun, Yu Cao, Rong Yang, Yingqiang Wei, Qiang Guo, You Ke, Maotao Yu, Yizheng Jin, Yang Liu, Qingqing Ding, Dawei Di, Le Yang, Guichuan Xing, He Tian, Chuanhong Jin, Feng Gao, Richard H. Friend, Jianpu Wang and Wei Huang, Perovskite light-emitting diodes based on solution-processed self-organized multiple quantum wells, Nature Photonics, 2016. 10(11), pp.699-+.

http://dx.doi.org/10.1038/NPHOTON.2016.185

Copyright: Nature Publishing Group

http://www.nature.com/

Postprint available at: Linköping University Electronic Press

http://urn.kb.se/resolve?urn=urn:nbn:se:liu:diva-133002

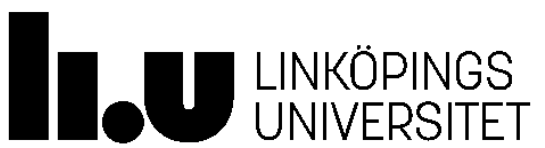


Perovskite Light-Emitting Diodes based on Solution-Processed, Self-Organised Multiple Quantum Wells

Nana Wang ${ }^{1 *}$ Lu Cheng ${ }^{1 *}$, Rui $\mathrm{Ge}^{1^{*}}$, Shuting Zhang ${ }^{1}$, Yanfeng Miao ${ }^{1}$, Wei Zou ${ }^{1}$, Chang $\mathrm{Yi}^{1}$, Yan Sun ${ }^{1}$, Yu Cao ${ }^{1}$, Rong Yang ${ }^{1}$, Yingqiang $\mathrm{Wei}^{1}$, Qiang Guo ${ }^{1}$, You $\mathrm{Ke}^{1}$, Maotao $\mathrm{Yu}^{1}$, Yizheng $\mathrm{Jin}^{2}$, Yang $\mathrm{Liu}^{3}$, Qingqing $\mathrm{Ding}^{4}$, Dawei $\mathrm{Di}^{5}$, Le $\mathrm{Yang}^{5}$, Guichuan Xing ${ }^{1}$, He Tian ${ }^{4}$, Chuanhong Jin ${ }^{4}$, Feng Gao ${ }^{6}$, Richard H. Friend ${ }^{5}$, Jianpu Wang $^{1}$ and Wei Huang ${ }^{1,7}$

${ }^{1}$ Key Laboratory of Flexible Electronics (KLOFE) \& Institute of Advanced Materials (IAM), Jiangsu National Synergetic Innovation Center for Advanced Materials (SICAM), Nanjing Tech University (NanjingTech), 30 South Puzhu Road, Nanjing 211816, China

${ }^{2}$ Center for Chemistry of High-Performance and Novel Materials, State Key Laboratory of Silicon Materials, and Department of Chemistry, Zhejiang University, Hangzhou 310027, China

${ }^{3}$ State Key Laboratory of Silicon Materials, Center for Chemistry of High-Performance and Novel Materials, and Department of Materials Science and Engineering, Zhejiang University, Hangzhou 310027, China

${ }^{4}$ Center of Electron Microscope, State Key Laboratory of Silicon Material, School of Material Science \& Engineering, Zhejiang University, Hangzhou 310027, China

${ }^{5}$ Cavendish Laboratory, Cambridge University, JJ Thomson Avenue, Cambridge, CB3 0HE, UK ${ }^{6}$ Biomolecular and Organic Electronics, IFM, Linköping University, Linköping 58183, Sweden ${ }^{7}$ Key Laboratory for Organic Electronics and Information Displays \& Institute of Advanced Materials (IAM), Jiangsu National Synergetic Innovation Center for Advanced Materials (SICAM), Nanjing University of Posts \& Telecommunications, 9 Wenyuan Road, Nanjing 210023, China

${ }^{*}$ These authors contributed equally to this work. 
Organometal halide perovskites can be processed from solutions at low temperatures to form crystalline direct-bandgap semiconductors with promising optoelectronic properties $^{1-5}$. However, the efficiency of their electroluminescence is limited by nonradiative recombination associated with defects and leakage current due to incomplete surface coverage ${ }^{6-9}$. Here we demonstrate a solution-processed perovskite light-emitting diode (LED) based on self-organised multiple quantum wells (MQWs) with excellent film morphology. The MQW-based LED exhibits a very high external quantum efficiency of up to $11.7 \%$, good stability and exceptional high-power performance with an energy conversion efficiency of 5.5\% at a current density of $100 \mathrm{~mA} \mathrm{~cm}^{-2}$. This outstanding performance arises because the lower bandgap regions which generate electroluminescence are effectively confined by perovskite MQWs with higher energy gaps, giving very efficient radiative decay. Surprisingly, there is no evidence that the large interfacial areas between different bandgap regions cause luminescence quenching.

Recently, high-efficiency photovoltaic devices based on three-dimensional (3D) organometal halide perovskites, such as $\mathrm{CH}_{3} \mathrm{NH}_{3} \mathrm{PbI}_{3}$ and $\mathrm{NH}_{2} \mathrm{CH}=\mathrm{NH}_{2} \mathrm{PbI}_{3}$ $\left(\mathrm{FAPbI}_{3}\right)$, have been demonstrated ${ }^{3-5}$. Hybrid 3D perovskites exhibit high photoluminescence quantum efficiencies (PLQEs) and good charge mobilities, making them also attractive for electroluminescence (EL) applications ${ }^{2,8,9}$. Encouraging performance metrics of light-emitting diodes (LEDs) based on 3D perovskites, such as low turn-on voltages and external quantum efficiencies (EQEs) of up to $3.5 \%$ at high current densities, have been demonstrated ${ }^{9}$. However, the EL quantum efficiency is far behind the limit predicated by $70 \%$ PLQE of the 3D perovskites, mainly due to the existence of current losses caused by incomplete surface coverage of the perovskite films and the fact that the high PLQE can only be obtained at high excitations ${ }^{8,9}$. By using thick $(>300 \mathrm{~nm})$ perovskite films, Cho et al. obtained LEDs with over 8\% $\mathrm{EQE}^{10}$. However, for this device, the turn-on voltage is high and the power efficiency is low, which may result from the thick perovskite layer used. In order to further enhance the performance of 3D perovskite-based LEDs, it is 
essential to obtain perovskite thin films with both complete surface coverage and high $\mathrm{PLQE}^{8-10}$. Moreover, device stability, which was proven to be a vital issue in organic-inorganic halide perovskite-based photovoltaics ${ }^{11}$, has not been addressed in perovskite LEDs.

The 3D perovskites are actually an extreme case of layered organometal halide perovskites with a general formula of $\mathrm{L}_{2}\left(\mathrm{SMX}_{3}\right)_{\mathrm{n}-1} \mathrm{MX}_{4}$, where $\mathrm{M}, \mathrm{X}, \mathrm{L}$, and $\mathrm{S}$ are a divalent metal cation, a halide, and organic cations with long and short chains, respectively (Fig. 1a) $)^{12-14}$. Here $\mathrm{n}$ is the number of semiconducting $\mathrm{MX}_{4}$ monolayer sheets within the two organic insulating layers (cation $L$ ), with $n=\infty$ corresponding to the structure of a $3 \mathrm{D}$ perovskite $\mathrm{SMX}_{3}$. With smaller numbers of $\mathrm{MX}_{4}$ layers, quantum confinement effects, such as an increase in bandgap and exciton energy, become important ${ }^{6,15}$. In consequence, the layered perovskites naturally form quantum-well structures. At the opposite extreme, when $n=1$, the layered perovskites form a monolayer structure of a two-dimensional (2D) perovskite $\mathrm{L}_{2} \mathrm{MX}_{4}$. The $2 \mathrm{D}$ $\mathrm{L}_{2} \mathrm{MX}_{4}$ perovskites generally have good film-formation properties ${ }^{13}$. Nevertheless, the PLQEs of the 2D perovskites are rather low at room temperature, owing to fast exciton quenching rates ${ }^{6,7}$. LEDs based on the 2D perovskites have been attempted, while the devices are either very low in efficiency or only operational at cryogenic temperatures $^{16-18}$. Here we demonstrate very efficient (up to $11.7 \%$ EQE) and high-brightness EL achievable at room temperature by using solution-processed perovskite multiple quantum wells (MQWs) with an energy cascade, which can combine the advantages of $2 \mathrm{D}$ and $3 \mathrm{D}$ perovskites. We note, a relevant perovskite LED work ${ }^{19}$ which shows a peak EQE of $8.8 \%$ has been published online during the revision of this paper.

A precursor solution of 1-naphthylmethylamine iodide (NMAI), formamidinium iodide (FAI), and $\mathrm{PbI}_{2}$ with a molar ratio of 2:1:2 dissolved in N,N-dimethylformamide (DMF) was used to deposit perovskite films (see Methods for details), which are abbreviated as $\mathrm{NFPI}_{7}$ films below. Atomic force microscopy 
(AFM) measurements show that the $\mathrm{NFPI}_{7}$ film has a smooth and uniform surface coverage (Fig. 1b). The root-mean-square roughness of the NFPI 7 film, $2.6 \mathrm{~nm}$, is comparable to that of the $2 \mathrm{D}(\mathrm{NMA})_{2} \mathrm{PbI}_{4}(\mathrm{n}=1)$ perovskite film, $1.4 \mathrm{~nm}$, and much lower than that of the 3D FAPbI $3(n=\infty)$ perovskite film, $18.8 \mathrm{~nm}$ (Supplementary Fig. S1).

We study the optical properties of the $\mathrm{NFPI}_{7}$ films. Because of the quantum confinement effects, the absorption and emission features of the layered perovskites depend on the value of $\mathrm{n}^{6,15}$. Previous studies on $\mathrm{PbI}_{4}$-based perovskites showed that the absorption peaks at $\sim 2.4, \sim 2.2, \sim 2.0$ and $\sim 1.9 \mathrm{eV}$ correspond to excitonic absorption of QWs with $n=1,2,3$ and 4 respectively ${ }^{6,20,21}$. Our results confirm that the (NMA) ${ }_{2} \mathrm{PbI}_{4}$ perovskite film, i.e. the $\mathrm{n}=1 \mathrm{QW}$ film, presents a strong peak at $2.43 \mathrm{eV}$ (Supplementary Fig. S2). The absorption spectrum of the NFPI film shows a strong exciton absorption peak at $2.18 \mathrm{eV}$ (Fig. 1c), indicating that the major component of the perovskite film is (NMA) $2\left(\mathrm{FAPbI}_{3}\right) \mathrm{PbI}_{4}$, i.e. the $\mathrm{n}=2 \mathrm{QWs}$. The shoulders at 2.43 and $1.95 \mathrm{eV}$ suggest the existence of small fractions of $\mathrm{n}=1$ and $\mathrm{n}=4$ perovskite QWs respectively, while the absorption peak of the $\mathrm{n}=3 \mathrm{QWs}$ at $\sim 2.0 \mathrm{eV}$ may be hidden by the absorption tail of the $n=2$ QWs. Optical features corresponding to QWs with higher $\mathrm{n}$ values are not evident in the absorption spectrum. They become visible in the PL spectrum, as shown by the dominating peak at $1.62 \mathrm{eV}$. This emission is $\sim 40 \mathrm{~nm}$ blue-shifted compared to that of bulk 3D $(n=\infty) F^{F A P b I}$ perovskite $(1.54 \mathrm{eV}$, Supplementary Fig. S2). The PL spectrum on a semi-log scale reveals several weak emission peaks at 2.38, 2.14 and $1.91 \mathrm{eV}$. With the absorption and emission spectra plotted in the same panel (Fig. 1c), one can tell that absorption and emission peaks for $\mathrm{n} \leq 4$ QWs correspond very well to each other. We point out that although $\mathrm{n}=2$ QWs give the strongest absorption peak in the $\mathrm{NFPI}_{7}$ films, emission from them is very weak.

The investigations above suggest that our NFPI7 film is an ensemble of self-organised multiple QWs with different exciton energies. The absorption and PL measurements 
clearly demonstrate energy transfer from QWs with large exciton energies to QWs with lower exciton energies. PL excitation measurements on the NFPI 7 film show that different emissions share a strong excitation peak at $2.18 \mathrm{eV}$ (Fig. 1d), showing that there is energy transfer from $n=2$ QWs to the lower energy emissions. For the $765 \mathrm{~nm} / 1.62 \mathrm{eV}$ emission, the PL excitation spectrum also reveals weak contributions from QWs with $n>2$. The observation of weak emission from the $n=1, n=2$ and $n=4$ QWs demonstrates that the energy migration is not complete. The cascade energy transfer of photon excitation in the perovskite MQWs is illustrated in Fig. 1e.

Transient absorption (TA) measurement further shows that the energy transfer between the perovskite QWs is an ultrafast process (Fig. 1f). With $400 \mathrm{~nm}$ excitation, the excitons are primarily formed in the $n=2$ QWs. This manifests as a fast build-up of the photobleaching $(\mathrm{PB})$ at the exciton absorption peak $(2.18 \mathrm{eV})$ of the $n=2 \mathrm{QWs}$. The evolution of the TA spectra shows that the excitons reach the large-n QWs ( 1.64 $\mathrm{eV}$ ) after some time delay. The PB of the large-n QWs resonance grows over time. Time traces at selected probe wavelengths are shown in Fig. 1g. The PB of $n=2$ QWs $(\sim 2.18 \mathrm{eV})$ shows an ultrafast dominant decay with a time constant of $\sim 0.5$ ps. This decay time is closely matched with the fast PB formation time of the large-n QWs $(\sim 1.64 \mathrm{eV})$. This result indicates that a substantial portion of the photo-generated excitons from $n=2$ QWs will be localised to large-n QWs within 0.5 ps. Subsequently, a slower decay of the $\mathrm{PB}$ of $\mathrm{n}=2$ QWs also matches well with a relative slower formation of the large-n QWs PB. This relatively slower exciton localisation time is fitted to be $\sim 50$ ps.

The rapid energy transfer process in the perovskite MQWs can essentially avoid the exciton quenching effect presented in previously reported 2D perovskites ${ }^{6,7}$. Figure $1 \mathrm{~h}$ shows the light-intensity-dependent PLQE of the NFPI7 film under a $445 \mathrm{~nm}$ cw laser excitation. The high PLQE of up to $60 \%$ suggests that the photogenerated excitons in low-n QWs can be energy transferred to the large-n QWs with superior emission properties, allowing efficient radiative recombination. Remarkably, the MQW films 
present high PLQEs at an excitation as low as $\sim 0.3 \mathrm{~mW} \mathrm{~cm}^{-2}$. This feature is very different from that of the bulk 3D perovskite films which have high PLQEs only at very high excitations, e.g. over $500 \mathrm{~mW} \mathrm{~cm}^{-2}$ (Supplementary Fig. S3 and ref. 2). High PLQEs of the perovskite emitters can be obtained when the nonradiative decay centres are filled by excitations ${ }^{9}$. In our MQW perovskite films, the exciton decay in large-n QWs is much slower (Supplementary Fig. S4) than the energy transfer process. As a result, most of the photo-generated excitons formed are concentrated to the small space of the large-n QWs, which can substantially increase the local exciton density in the emissive large-n QWs. Furthermore, the QW structure can effectively confine the excitons and reduce the exciton diffusion lengths, which can decrease the exciton-defect quenching probability.

The desirable film morphology and optical properties of the NFPI film encourage us to fabricate LEDs based on perovskite MQWs. Figure 2a shows the cross-sectional view of a device with a multi-layer structure of indium tin oxide (ITO)/polyethylenimine ethoxylated (PEIE)-modified zinc oxide (ZnO, $\sim 20 \mathrm{~nm}$ )/perovskite MQWs ( 30 nm)/poly(9,9-dioctyl-fluorene-co-N-(4-butylphenyl)diphenylamine) (TFB, $\sim 40 \mathrm{~nm}$ )/molybdenum oxide $\left(\mathrm{MoO}_{\mathrm{x}}, \sim 7 \mathrm{~nm}\right.$ )/gold (Au, $\left.\sim 60 \mathrm{~nm}\right)$. All layers, apart from the $\mathrm{MoO}_{\mathrm{x}} / \mathrm{Au}$ electrode, were deposited from solutions. The flat-band energy levels of the layers are indicated in Fig. 2b, and the energy level values for $\mathrm{NFPI}_{7}$ film were estimated by UPS (Supplementary Fig. S5) and optical measurements. The PEIE-modified $\mathrm{ZnO}$ and TFB layers are employed as electron-transporting interlayers and hole-transporting interlayers respectively. The $\mathrm{MoO}_{\mathrm{x}} / \mathrm{Au}$ bilayers and ITO are selected as top electrodes and bottom electrodes respectively.

A cross-sectional sample of the NFPI 7 device was analysed by an aberration-corrected scanning transmission electron microscope (STEM). A low-magnification high-angle annular dark-field (HAADF) image (Fig. 2a) illustrates the multiple layers of materials with distinct contrast. The $\sim 30 \mathrm{~nm}$ thick perovskite layer is smooth and 
pin-hole free based on through STEM inspections, agreeing well with the AFM results that the $\mathrm{NFPI}_{7}$ film has a low surface roughness. Zoomed-in observations indicate that the perovskite layer exhibits contrasts, with the majority of brighter regions close to the perovskite/TFB interface. We propose that the contrasts in the perovskite layer reflect the spatial distributions of QWs with different n values. Perovskite QWs with larger $\mathrm{n}$ values accommodate more $\mathrm{PbI}_{4}$ units per volume, thereby result in a higher intensity of large-angle elastic scattering and in consequence appear brighter in the HAADF imaging. In other words, the majority of large-n QWs are located in the regions close to the perovskite/TFB interface. This deduction is supported by both energy-dispersive X-ray spectroscopy (EDX) elemental mapping and high-resolution transmission electron microscopy (HRTEM) results (Figs. 2c, d). EDX maps show stronger signals of both lead and iodide in the brighter regions. Distribution of lead and iodide across the perovskite layer suggests that the large-n QWs and low-n QWs locate at the interface of perovskite/TFB and perovskite/ZnO respectively. The large-n QWs region is much narrower than the low-n QWs region which presumably is the $\mathrm{n}=2$ QWs, and a gradually graded or mixed QWs region is in between. HRTEM and the corresponding Fast Fourier Transformation (FFT) analyses (Fig. 2d and Supplementary Fig. S6) show that the perovskite crystals close to the perovskite/TFB interface share the same cubic structure with the $3 \mathrm{D} \alpha-\mathrm{FAPbI}_{3}{ }^{22}$.

Figure 3a shows the EL spectra of devices based on NFPI7 MQW films (see Supplementary Fig. S7 for characterization setup). The EL emission peak at near-infrared (NIR), $786 \mathrm{~nm} / 1.58 \mathrm{eV}$, is $\sim 20 \mathrm{~nm}$ blue-shifted compared with the EL from bulk 3D FAPbI3 perovskites (Supplementary Fig. S8). The shapes of the EL spectra do not change at different bias voltages (Supplementary Fig. S9). The NFPI 7 -MQW LED turns on at a low voltage of $1.5 \mathrm{~V}$ (Fig. 3b). The current density and radiance rise rapidly by several orders of magnitude after turn-on, implying excellent charge injection and transport in the MQW LEDs. The EQE of the LED reaches $9.6 \%$ at $2.3 \mathrm{~V}$ with a current density of $15 \mathrm{~mA} \mathrm{~cm}^{-2}$ (Fig. 3c). A high radiance of $55 \mathrm{~W} \mathrm{sr}^{-1} \mathrm{~m}^{-2}$ is achieved at $3.6 \mathrm{~V}$. The angular emission intensity of the device 
follows a Lambertian profile (Supplementary Fig. S10). The statistics from 67 devices present an average EQE of $7.0 \%$ with a relative standard deviation of $18 \%$ (Fig. 3d), demonstrating good reproducibility of the NFPI7-MQW LEDs.

We consider that the superior EL performance in the MQW LEDs is due to the confinement of injected charges in the large-n QWs which have excellent emission properties as shown by the PL measurements. In EL device operation, electrons and holes are injected into the perovskite layer and accumulated at the large-n QWs because of the cascade energy structure of the perovskite MQW films. In other words, the injected charges can directly recombine at the very thin layer of large-n QWs. This is consistent with our finding that the weak emissions at higher energies observed in the PL spectrum are absent in the EL measurements and the EL emission peak is slightly red-shifted compared to the main PL peak (Fig. 3a and Fig. 1c). As shown by the PL investigations (Fig. 1h), the very thin layer of large-n QWs is highly emissive even at low excitations, which results in the high EL efficiency in our MQW perovskite LEDs. In order to fabricate high-efficiency perovskite LEDs, it is a challenge to obtain a large-n emissive thin layer with good coverage sandwiched between two non-quenching contacts with appropriate energy levels, while our MQW structure provides a simple alternative solution to it.

One of the advantages for perovskite materials is that the optoelectronics properties can be easily tuned by adjusting the compositions. In perovskite solar cells, it has been shown that the device performance can be optimised by using mixed halogens which improves crystallinity of the perovskite film ${ }^{23}$. We show that it is possible to further enhance the efficiency of MQW LEDs by adopting a similar strategy (Supplementary Table S1). For example, we used a precursor solution of NMAI: FABr: PbI2 with a molar ratio of 2:1:2 in DMF to deposit new perovskite MQW films (abbreviated as NFPI ${ }_{6} \mathrm{~B}$ below). This NFPI ${ }_{6} \mathrm{~B}$ film has a PLQE of $67 \%$ (Supplementary Fig. S3), higher than that of the NFPI7 film. The EL emission peak of LEDs based on the NFPI 6 B film is shifted to $763 \mathrm{~nm}$ (Fig. 3a). The champion 
$\mathrm{NFPI}_{6} \mathrm{~B}$ LED turns on at $1.3 \mathrm{~V}$. The EQE reaches $11.7 \%$ at $2.6 \mathrm{~V}$ with a current density of $38 \mathrm{~mA} \mathrm{~cm}^{-2}$. A high radiance of $\sim 82 \mathrm{~W} \mathrm{sr}^{-1} \mathrm{~m}^{-2}$ is achieved at $3.6 \mathrm{~V}$. These results represent the highest EQE for perovskite-based LEDs $^{9}$. Remarkably, the device is highly efficient at large current densities. The energy conversion efficiency (wall-plug efficiency) of our $\mathrm{NFPI}_{6} \mathrm{~B}$ MQW LED is $5.5 \%$ at $100 \mathrm{~mA} \mathrm{~cm}{ }^{-2}$, which is more efficient than the state-of-the-art vacuum-deposited organic LEDs at this high current density (Supplementary Table S2) ${ }^{24-26}$. The EQE histogram for 70 NFPI 6 B-MQW LEDs shows an average peak EQE of $8.8 \%$ and a small relative standard deviation of $11 \%$ (Fig. 3d). Owing to the good film uniformity, the size of the perovskite MQW LEDs can be easily scaled up by adopting the same device structure and fabrication process. Figure 3a also shows uniform and bright EL emission from a $\sim 64 \mathrm{~mm}^{2}$ NFPI ${ }_{6} B-M Q W$ device with a peak EQE of 7.5\%.

We can tune the EL across a wide range of the spectrum by further changing the halide compositions in precursor solutions. For example, the perovskite MQW LEDs can achieve narrow emission peaks at 736, 685, 664, 611 or $518 \mathrm{~nm}$, when the MQW films are produced by using precursor solutions with various ratios of $\mathrm{Br}$ to I (Fig. 4a). The various combinations of precursor solutions to produce the $\mathrm{NFPI}_{\mathrm{y}} \mathrm{B}_{7-\mathrm{y}}$ ( $\mathrm{y}$ represents the fraction of I) films are provided in Supplementary Table S1. Figure 4b demonstrates bright and uniform emission from the green and red MQW LEDs, respectively. The symmetric and narrow emission peaks at $518 \mathrm{~nm}$ and $685 \mathrm{~nm}$ correspond to Commission Internationale de l'Eclairage (CIE) colour coordinates of $(0.11,0.74)$ and $(0.72,0.28)$ respectively, which are ideal for display applications. Supplementary Table S1 also summarises the device performance of different-colour MQW LEDs with the same device structure. The performance of the visible LEDs is inferior to that of the NIR devices, which is likely due to either inefficient charge injection to the emitters with larger band gap or lower PL efficiencies. By further optimising the device structure and film processing, it is possible to enhance the MQW LED device performance in the visible range. 
In addition to the remarkably high efficiency and readily colour tunability, the devices based on perovskite MQWs also demonstrate reasonably good stability. As shown in Fig. 4c, the EQE of the $\mathrm{NFPI}_{6} \mathrm{~B}$ devices dropped to half of the initial value after $\sim 2 \mathrm{~h}$ under a constant current density of $10 \mathrm{~mA} \mathrm{~cm}^{-2}$. This result shows that the stability of perovskite MQW LEDs is significantly improved (by two orders of magnitude) compared with LEDs based on the 3D perovskites produced in our laboratory, which can only survive $\sim 1$ minute under similar conditions (Supplementary Fig. S8). We believe both the high power conversion efficiency of the LED and the stability of the perovskites QW films ${ }^{21}$ contribute to the improved device lifetime.

The use of semiconductors with different bandgaps to engineer high performance optoelectronic devices has of course been very effectively developed in III-V semiconductors. This allows control of charge recombination sites and luminescence wavelength, but it requires very close control of lattice parameter matching to avoid electronic defects that cause trapping and quenching. Our findings reveal that these mixed energy gap perovskite structures show no evidence for higher electronic defect densities than single gap materials, in spite of the very high interfacial areas that must be present. This fact provides real promise for the further development of this class of materials, particularly for light emission. 


\section{Acknowledgements}

This work is financially supported by the National Basic Research Program of ChinaFundamental Studies of Perovskite Solar Cells (2015CB932200), the Natural Science Foundation of Jiangsu Province, China (BK20131413, BK20140952, BM2012010), the National Natural Science Foundation of China (11474164, 51522209, 61405091, 11474249), the National 973 Program of China (2015CB654901), the Jiangsu Specially-Appointed Professor program, the Synergetic Innovation Center for Organic Electronics and Information Displays, the Fundamental Research Funds for the Central Universities (2015FZA3005), the China Postdoctoral Science Foundation, the Swedish Government Strategic Research Area in Materials Science on Functional Materials at Linköping University (Faculty Grant SFO-Mat-LiU \# 2009-00971), the Swedish Research Council (VR, 330-2014-6433), and the European Commission Marie Skłodowska-Curie actions (691210 and INCA 600398). We thank H. Li for the assistance of the AFM measurement, C. Wang for the assistance of the UV-vis absorbance measurement, $\mathrm{H}$. He \& B. Su for the assistance of the PLE and TCSPC measurement, and X. Liang for the UPS measurement. We thank P. Fowler for proof reading and X. Liu for helpful discussions.

\section{Materials \& Correspondence}

Authors to whom correspondence and requests for materials: Prof. Jianpu Wang (iamjpwang@njtech.edu.cn) or Prof. Wei Huang (wei-huang@njtech.edu.cn)

\section{Author Contributions}

J.W. had the idea for and designed the experiments. J.W. and W.H. supervised the work. L.C., R.G., N.W. and S.Z. carried out the device fabrication and characterizations. Y.M., Y.S. and Y.C. conducted the optical measurements. W.Z. managed to set up the testing systems and took part in the optical measurements. C.Y. and Y.C. synthesized the NMAI/NMABr and measured AFM. R.Y., Q.G., Y.K., M.Y., D.D. and L.Y. participated in device fabrication and characterizations. G.X. measured transient absorption. Y.L., Q.D., H.T, C.J., Y.J. and Y.W. carried out HRTEM and 
STEM characterizations. J.W., N.W. and F.G. wrote the first draft of the manuscript. Y.J., R.H.F. and W.H. participated in data analysis and provided major revisions. All authors discussed the results and commented on the manuscript.

\section{Competing financial interests}

The authors declare no competing financial interests. 


\section{Figures:}

Figure 1

a

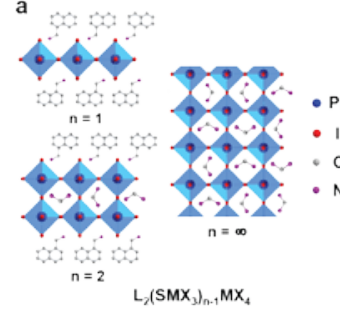

e

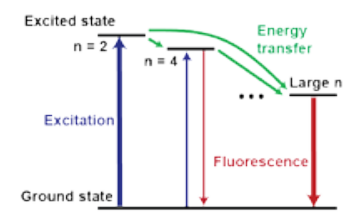

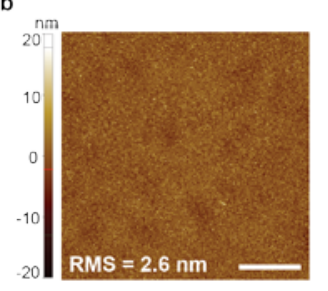

f

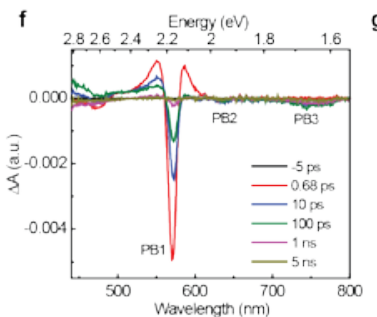

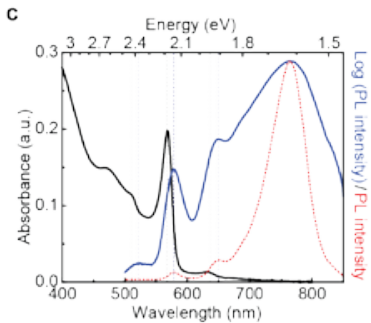

g

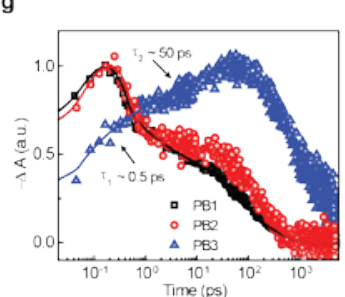

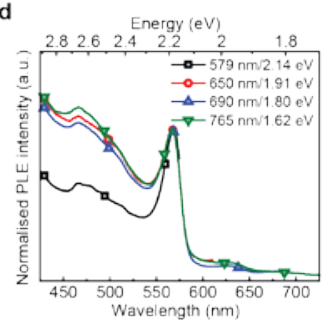

h

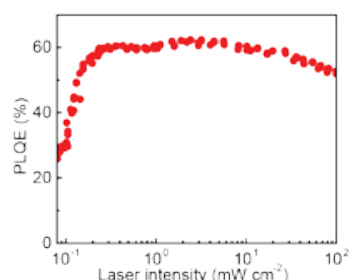

Figure 1. Perovskite MQW films. a, Schematic representation of the structures of the layered lead halide perovskites with $n=1, n=2$ and $n=\infty$. b. An AFM height image of the NFPI 7 MQW film (scale bar: $5 \mu \mathrm{m}$ ). c, Absorption and PL (445 nm excitation) spectra of the NFPI 7 MQW film deposited onto quartz substrates. The PL spectrum is plotted in log scale (blue curve) and linear scale (red curve). The three pairs of the dashed lines (from left to right) correspond to the absorption (black) and PL (red) peaks from the QWs with n=1, 2 and 4, respectively. d, PL excitation spectra of the NFPI7 MQW film at various emission energies. a.u., arbitrary units. e, Schematic of cascade energy transfer in MQWs. Excitation energy is transferred downstream from lower-n QWs to larger-n QWs, and the emission is mainly from larger-n QWs. f, TA spectra of the MQWs at selected probe delay times. g, Normalised bleaching kinetics at $2.18 \mathrm{eV}$ (black Square), $1.95 \mathrm{eV}$ (red Circle) and $1.64 \mathrm{eV}$ (blue Triangular) for the MQWs following excitation at $400 \mathrm{~nm}\left(1 \mathrm{KHz}, \quad 50 \mathrm{fs}, \quad \sim 0.4 \mu \mathrm{J} \mathrm{cm}^{-2}\right) . \quad$ h, Excitation-intensity-dependent PLQE of the NFPI7 MQW film. 


\section{Figure 2}
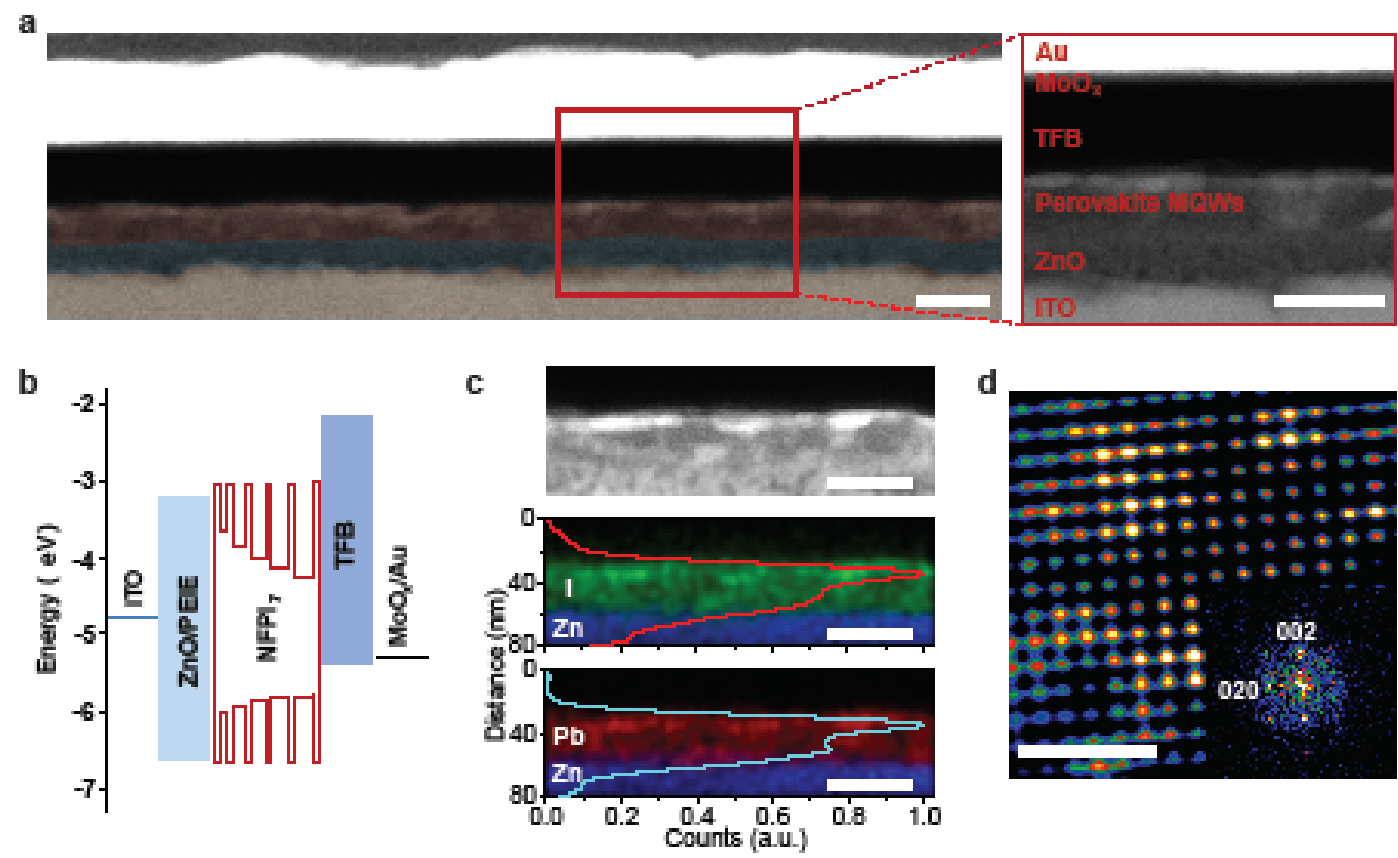

Figure 2. Device structure of the $\mathrm{NFPI}_{7}$ perovskite MQW LED. a, A cross-sectional scanning transmission electron microscopy (STEM) image (scale bar: $50 \mathrm{~nm})$ showing the device architecture: ITO/PEIE modified ZnO ( $20 \mathrm{~nm}) / \mathrm{NFPI}_{7}$ $(\sim 30 \mathrm{~nm}) / \mathrm{TFB}(\sim 40 \mathrm{~nm}) / \mathrm{MoO}_{\mathrm{x}}(\sim 7 \mathrm{~nm}) / \mathrm{Au}$. The different layers in the left panel have been tinted. b, Flat-band energy level diagram. Except $\mathrm{NFPI}_{7}$, energy level values of other layers were taken from the literature ${ }^{9}$. c, EDX mapping. Color-mixed EDX mapping images (scale bar: $50 \mathrm{~nm}$ ) present the element distribution of lead (Red), iodide (Green) and zinc (Blue). Normalised EDX counts distribution of lead and iodide across the perovskite layer are also presented. d, Fast-Fourier-transform-filtered HRTEM image (scale bar: $2 \mathrm{~nm}$ ) and FFT analyses (inset) of a perovskite crystal close to the TFB/perovskite interface show that this crystal has a cubic structure similar to that of the $3 \mathrm{D} \alpha-\mathrm{FAPbI} 3^{22}$. 


\section{Figure 3}

a

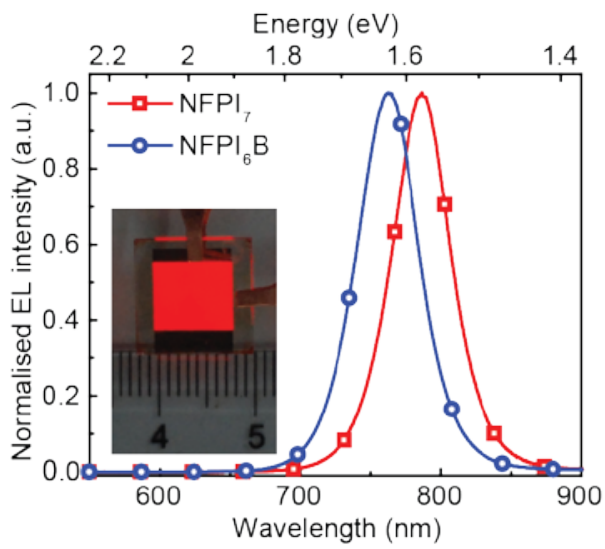

C

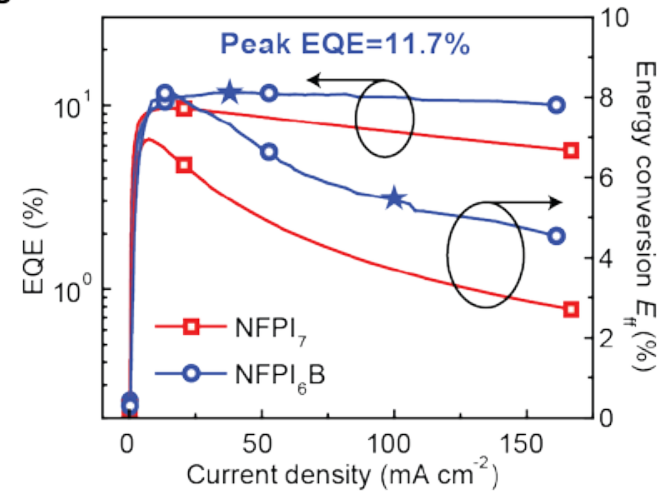

b

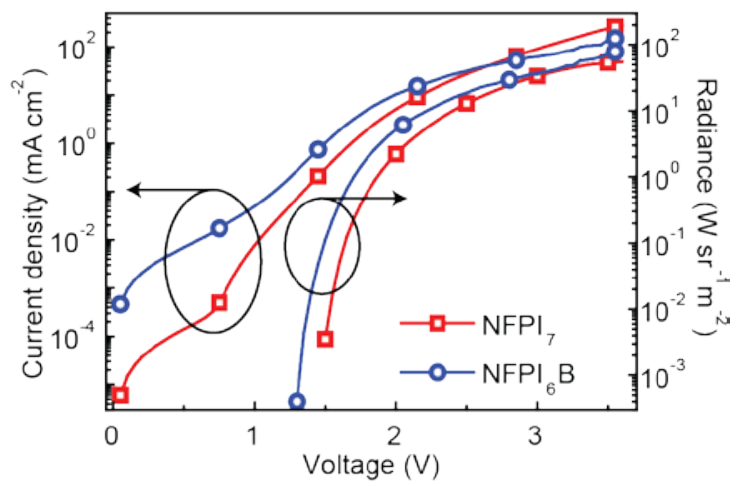

d

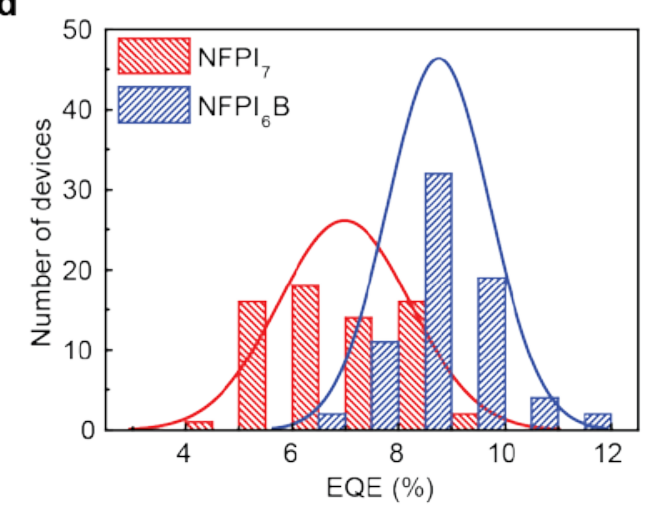

Figure 3. Optoelectronic characteristics of the $\mathrm{NFPI}_{7}$ and $\mathrm{NFPI}_{6} \mathrm{~B}$ perovskite MQW LEDs. a, EL spectra of the devices under 3 V. Inset: a photograph of a 64-mm² NFPI 6 B device. b, Dependence of current density and radiance on the driving voltage. Radiance of $55 \mathrm{~W} \mathrm{sr}^{-1} \mathrm{~m}^{-2}$ and $82 \mathrm{~W} \mathrm{sr}^{-1} \mathrm{~m}^{-2}$ are obtained under $3.6 \mathrm{~V}$ for NFPI7 and NFPI ${ }_{6} \mathrm{~B}$ devices, respectively. c, EQE and energy conversion efficiency versus current density. For the NFPI ${ }_{6} \mathrm{~B}$ MQW LED, a peak EQE of $11.7 \%$ is achieved at a current density of $38 \mathrm{~mA} \mathrm{~cm}^{-2}$ and a energy conversion efficiency of $5.5 \%$ is obtained at a current density of $100 \mathrm{~mA} \mathrm{~cm}^{-2}$. d, Histograms of peak EQEs measured from 67 NFPI 7 and 70 NFPI 6 B devices. 


\section{Figure 4}

a

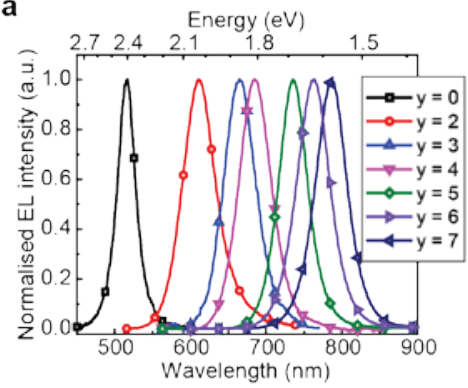

b

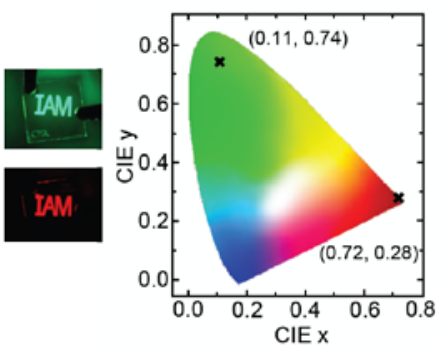

C

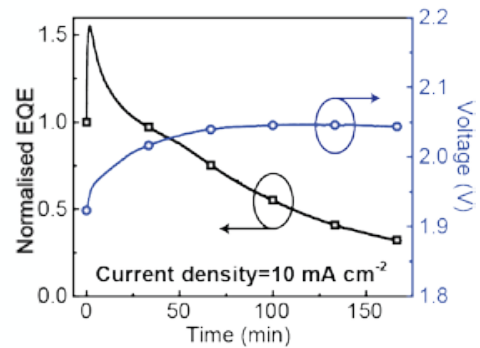

Figure 4. EL color tunability and device stability of the perovskite MQW LEDs.

a, EL spectra of MQW LEDs based on the $\mathrm{NFPI}_{\mathrm{y}} \mathrm{B}_{7-\mathrm{y}}$ films, $\mathrm{y}=0$, 2, 3, 4, 5, 6 and 7, respectively, which are processed using various combinations of precursor solutions of NMAI (NMABr), FAI (FABr) and $\mathrm{PbI}_{2}\left(\mathrm{PbBr}_{2}\right)$ (refer to Supplementary Table S1 for details). b, Photographs of green and red MQW LEDs with the logo of the Institute of Advanced Materials (IAM), and the corresponding CIE coordinates. c, Stability data for a NFPI 7 EL device tested at a constant current density of $10 \mathrm{~mA} \mathrm{~cm}^{-2}$. 


\section{Methods}

Synthesis and materials preparation. NFPI7 precursor solutions were prepared by dissolving NMAI, FAI and $\mathrm{PbI}_{2}$ with a molar ratio of 2:1:2 in DMF (10 wt.\%) and stirred at $60{ }^{\circ} \mathrm{C}$ for $2 \mathrm{~h}$ in a nitrogen-filled glovebox. The NMAI was synthesized by adding $4.34 \mathrm{~g}$ hydroiodic acid (45 wt.\% in water) to a stirring solution of 1-naphthalenemethylamine $(12.72 \mathrm{mmol})$ in tetrahydrofuran (THF, $50 \mathrm{~mL})$ at $0{ }^{\circ} \mathrm{C}$ for $2 \mathrm{~h}$. The solution was then evaporated at $50{ }^{\circ} \mathrm{C}$ to obtain the NMAI precipitate, which was washed three times with THF: $\mathrm{CH}_{2} \mathrm{Cl}_{2}$ (3:1) mixture and then dried under vacuum. NMABr was synthesized by using similar method with the hydroiodic acid substituted by the hydrobromic acid. $\mathrm{NFPI}_{\mathrm{y}} \mathrm{B}_{7-\mathrm{y}}$ precursor solutions were prepared by altering composition of NMAI (NMABr), FAI (FABr), and $\mathrm{PbI}_{2}\left(\mathrm{PbBr}_{2}\right)$ with a molar ratio of 2:1:2 in DMF (10 wt.\%), except for $\mathrm{NFPI}_{2} \mathrm{~B}_{5}$ from a precursor solution with a molar ratio of 2:1:1:1 in DMF (10 wt.\%) of NMABr:FABr:PbBr2:PbI 2 .

(NMA) ${ }_{2} \mathrm{PbI}_{4}$ precursor solutions were prepared by dissolving NMAI and $\mathrm{Pbl}_{2}$ with molar ratio of 1:1 in DMF (10 wt.\%) and stirred at $60{ }^{\circ} \mathrm{C}$ for $2 \mathrm{~h}$ in a glovebox.

Colloidal ZnO nanocrystals were synthesized by a solution-precipitation process and the details can be found in the literature ${ }^{9}$.

Device fabrication. Solutions of $\mathrm{ZnO}$ nanocrystals were spin-coated onto the ITO-coated glass substrates at 4,000 rpm for $45 \mathrm{~s}$ and annealed in air at $150{ }^{\circ} \mathrm{C}$. Then the substrates were transfered into a glovebox. Next, a solution of PEIE in 2-methoxyethanol (0.4 wt.\%) was spin-coated onto the $\mathrm{ZnO}$ films at a speed of 5,000 rpm. The substrates were rinsed twice with DMF, leaving ultrathin layers of PEIE on top of the ZnO films. The ultrathin layer of PEIE can significantly decrease the work function of $\mathrm{ZnO}^{27}$. The perovskite films were prepared by spin coating the precursor solution onto the PEIE treated ZnO films, followed by annealing on a hot plate at $100{ }^{\circ} \mathrm{C}$. The TFB layers were deposited from an m-xylene solution $\left(8 \mathrm{mg} \mathrm{mL}^{-1}\right.$ ) at 2,000 rpm. Finally, the $\mathrm{MoO}_{\mathrm{x}} / \mathrm{Au}$ electrode was deposited using a thermal evaporation system through a shadow mask under a base pressure of $\sim 6 \times 10^{-7}$ Torr. The device area was $3 \mathrm{~mm}^{2}$ as defined by the overlapping area of the ITO films and top electrodes. 
Characterization. All perovskite LED device characterizations were carried out at room temperature in a nitrogen-filled glovebox. A Keithley 2400 source meter and a fiber integration sphere (FOIS-1) couple with a QE65 Pro spectrometer was used for the measurements ${ }^{28}$. The LED devices are tested on top of the integration sphere and only forward light emission can be collected, which is consistent with the standard OLED characterization method ${ }^{29}$. Figure S9 shows the layout of our device characterization setup. The devices were swept from zero bias to forward bias. The time evolution of EQEs and voltages were measured using the same testing system. The angular dependence of emission intensity was measured by using a Thorlabs PDA100A detector with a fixed distance of $200 \mathrm{~mm}$ away from the EL device. To verify the accuracy of the measurement setup, we have cross-checked the performance of two sets of perovskite LEDs prepared under identical conditions but separately measured at Nanjing Tech University and University of Cambridge. The results obtained in the two different labs are in good agreement.

AFM images were collected in non-contact mode (Park XE7). The STEM images and EDX elemental mapping of the perovskite films were made on a FEI Titan G2 80-200 ChemiSTEM operated at $200 \mathrm{keV}$. An element determination with outstanding sensitivity can be provided by ChemiSTEM EDX system. The HRTEM images were collected on a Tecnai G2 F20 microscope operated at $200 \mathrm{keV}$. The cross-sectional samples were prepared by using duel beam focused-ion-beam equipment (FEI Quata 3D FEG).

UV-vis absorbance spectra were recorded on a UV-vis spectrophotometer with an integrating sphere (Cary 5000, Agilent). PL spectra of the perovskite films were measured at room temperature using a fluorescent spectrophotometer (F-4600, HITACHI) with a $200 \mathrm{~W}$ Xe lamp as an excitation source. The excitation spectra and time resolved fluorescence spectra of the perovskite films were obtained by using an Edinburgh Instruments (FLS920) spectrometer. For the time-resolved PL measurements, the perovskite films were excited from the quartz glass substrate side

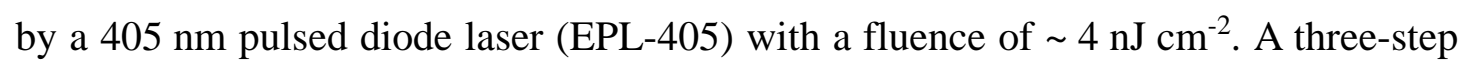
technique was used to obtain PLQE of perovskite films by combination of a $445 \mathrm{~nm}$ 
CW laser, optical fiber, spectrometer and integrating sphere ${ }^{30}$.

For transient absorption measurements, the laser source was a Coherent LibraTM regenerative amplifier (50 fs, $1 \mathrm{KHz}, 800 \mathrm{~nm}$ ) seeded by a Coherent VitesseTM oscillator (50 fs, $80 \mathrm{MHz}$ ). $800 \mathrm{~nm}$ wavelength laser pulses were from the regenerative amplifier while $400 \mathrm{~nm}$ wavelength laser pulses were obtained with a BBO doubling crystal. Broad band femtosecond transient absorption spectra of the perovskite films were taken using the Ultrafast System HELIOSTM transient absorption spectrometer. The broadband probe pulses $(420-800 \mathrm{~nm})$ were generated by focusing a small portion $(\sim 10 \mu \mathrm{J})$ of the fundamental $800 \mathrm{~nm}$ laser pulses into a $2 \mathrm{~mm}$ sapphire plate. The thickness of the perovskite films is $\sim 30 \mathrm{~nm}$ (same as in EL devices) for the above optical characterizations.

UPS spectra were collected on a Thermo ESCALAB-250Xi spectrometer with an applied bias of $-10 \mathrm{~V}$. He I UV radiation source $(21.2 \mathrm{eV})$ was used. The overall resolution of the instrument is $0.1 \mathrm{eV}$. 


\section{References:}

1. Xing, G. et al. Low-temperature solution-processed wavelength-tunable perovskites for lasing. Nat. Mater. 13, 476-480 (2014).

2. Deschler, F. et al. High photoluminescence efficiency and optically pumped lasing in solution-processed mixed halide perovskite semiconductors. J. Phys. Chem. Lett. 5, 1421-1426 (2014).

3. Stranks, S. D. et al. Electron-hole diffusion lengths exceeding 1 micrometer in an organometal trihalide perovskite absorber. Science 342, 341-344 (2013).

4. Burschka, J. et al. Sequential deposition as a route to high-performance perovskite-sensitized solar cells. Nature 499, 316-319 (2013).

5. Yang, W. S. et al. High-performance photovoltaic perovskite layers fabricated through intramolecular exchange. Science 348, 1234-1237 (2015).

6. Hong, X., Ishihara, T. \& Nurmikko, A. V. Dielectric Confinement effect on excitons in PbI4-based layered semiconductors. Phys. Rev. B 45, 6961-6964 (1992).

7. Gauthron, K. et al. Optical spectroscopy of two-dimensional layered $\left(\mathrm{C}_{6} \mathrm{H}_{5} \mathrm{C}_{2} \mathrm{H}_{4}-\mathrm{NH}_{3}\right)_{2}-\mathrm{PbI}_{4}$ perovskite. Opt. Express 18, 5912-5919 (2010).

8. Tan, Z.-K. et al. Bright light-emitting diodes based on organometal halide perovskite. Nat. Nanotechnol. 9, 687-692 (2014).

9. Wang, J. et al. Interfacial control toward efficient and low-voltage perovskite light-emitting diodes. Adv. Mater. 27, 2311-2316 (2015).

10. Cho, H. et al. Overcoming the electroluminescence efficiency limitations of 
perovskite light-emitting diodes. Science 350, 1222-1225 (2015).

11. Li, X. et al. Improved performance and stability of perovskite solar cells by crystal crosslinking with alkylphosphonic acid $\omega$-ammonium chlorides. Nat. Chem. 7, 703-711 (2015).

12. Calabrese, J. et al. Preparation and characterization of layered lead halide compounds. J. Am. Chem. Soc. 113, 2328-2330 (1991).

13. Mitzi, D. B., Chondroudis, K. \& Kagan, C. R. Organic-inorganic electronics. IBM J. Res. Dev. 45, 29-45 (2001).

14. Tyagi, P., Arveson, S. M. \& Tisdale, W. A. Colloidal organohalide perovskite nanoplatelets exhibiting quantum confinement. J. Phys. Chem. Lett. 6, 1911-1916 (2015).

15. Ishihara, T. Optical-properties of PbI-based perovskite structures. J. Lumin. 60-61, 269-274 (1994).

16. Hong, X., Ishihara, T. \& Nurmikko, A. V. Photoconductivity and electroluminescence in lead iodide based natural quantum-well structures. Solid State Commun. 84, 657-661 (1992).

17. Era, M., Morimoto, S., Tsutsui, T. \& Saito, S. Organic-inorganic heterostructure electroluminescent device using a layered perovskite semiconductor $\left(\mathrm{C}_{6} \mathrm{H}_{5} \mathrm{C}_{2} \mathrm{H}_{4} \mathrm{NH}_{3}\right)_{2} \mathrm{PbI}_{4}$. Appl. Phys. Lett. 65, 676-678 (1994).

18. Chondroudis, K. \& Mitzi, D. B. Electroluminescence from an organic-inorganic perovskite incorporating a quaterthiophene dye within lead halide perovskite layers. Chem. Mater. 11, 3028-3030 (1999). 
19. Yuan, M. et al. Perovskite energy funnels for efficient light-emitting diodes. Nat. Nanotechnol. doi:10.1038/nnano.2016.110, (2016).

20. Tanaka, K. \& Kondo, T. Bandgap and exciton binding energies in lead-iodide-based natural quantum-well crystals. Sci. Technol. Adv. Mater. 4, 599-604 (2003).

21. Smith, I. C., Hoke, E. T., Solis-Ibarra, D., McGehee, M. D. \& Karunadasa, H. I. A layered hybrid perovskite solar-cell absorber with enhanced moisture stability. Angew. Chem.-Int. Ed. 53, 11232-11235 (2014).

22. Weller, M. T., Weber, O. J., Frost, J. M. \& Walsh, A. Cubic perovskite structure of black formamidinium lead iodide, $\alpha$-[HC( $\left.\left(\mathrm{NH}_{2}\right)_{2}\right] \mathrm{PbI}_{3}$, at 298 K. J. Phys. Chem. Lett. 6, 3209-3212 (2015).

23. Jeon, N. J. et al. Compositional engineering of perovskite materials for high-performance solar cells. Nature 517, 476-480 (2015).

24. Graham, K. R. et al. Extended conjugation platinum(II) porphyrins for use in near-infrared emitting organic light emitting diodes. Chem. Mater. 23, 5305-5312 (2011).

25. Helander, M. G. et al. Chlorinated indium tin oxide electrodes with high work function for organic device compatibility. Science 332, 944-947 (2011).

26. Lai, C.-C. et al. m-indolocarbazole derivative as a universal host material for RGB and white phosphorescent OLEDs. Adv. Funct. Mater. 25, 5548-5556 (2015).

27. Zhou, Y. et al. A universal method to produce low-work function electrodes for 
organic electronics. Science 336, 327-332 (2012).

28. Dai, X. et al. Solution-processed, high-performance light-emitting diodes based on quantum dots. Nature 515, 96-99 (2014).

29. Forrest, S. R., Bradley, D. D. C. \& Thompson, M. E. Measuring the efficiency of organic light-emitting devices. Adv. Mater. 15, 1043-1048 (2003).

30. De Mello, J. C., Wittmann, H. F. \& Friend, R. H. An improved experimental determination of external photoluminescence quantum efficiency. Adv. Mater. 9, 230-232 (1997). 